\title{
GFF Classifier for Detection of Diabetic Retinopathy in Retinal Images
}

\author{
Amol Prataprao Bhatkar \\ Assistant Professor \\ Department of Electronics and Telecomm \\ Engineering \\ Anuradha Engineering College \\ Chikhli, Buldana (M.S.) INDIA
}

\author{
G. U. Kharat, $\mathrm{PhD}$ \\ Principal \\ Sharadchandra Pawar College of Engineering, \\ Otur, \\ Pune(M.S.) INDIA
}

\begin{abstract}
The emerging situation in today's world suggests diabetic retinopathy may be a major problem in the medical world. Diabetic retinopathy is dangerous because it cannot be identified in its earlier stages and leads to vision loss. Hence, detection of diabetic retinopathy in early stage is very much important. This paper focuses on Generalized Feed Forward Neural Network (GFFNN) to detect diabetic retinopathy in retinal images. In this paper the authors present the GFFNN as a classifier to classify retinal images as normal and abnormal. 64-point Discrete Cosine Transform (DCT) and 09 statistical parameters such as Entropy, Mean, Standard deviation, Average, Euler number, Contrast, Correlation, Energy and Homogeneity are extracted from fundus retinal images to form a feature vector. The feature vector is used to train and test the GFFNN. The training and cross validation recognition rate by the GFFNN are $100 \%$ and $95.45 \%$ respectively for detection of normal and abnormal retinal images.
\end{abstract}

\section{General Terms}

Neural Network, Classification of retinal images

\section{Keywords}

Generalized Feed Forward Neural Network (GFFNN), Discrete Cosine Transform (DCT), Fundus retinal images database DIARETDB0.

\section{INTRODUCTION}

Blood vessels throughout the body, particularly in the kidneys and eyes get affected in diabetes. When blood vessels in the eye are affected, the condition is referred to as diabetic retinopathy (DR). The major public health problem which is a leading cause of blindness in the World is diabetic retinopathy. It is caused by changes in the blood vessels of the retina. Diabetic retinopathy becomes symptomatic in its later stage. In the first stage, diabetic patients may not be aware of having infected by the disease [2]. To avoid vision loss early detection of diabetic retinopathy is very important. Ophthalmologist can obtain retinal images from patients to be diagnosed by using a fundus camera. From the image, symptoms will be identified manually by an ophthalmologist. Therefore more time is required to diagnose more number of patients. A computerized screening system can be used for fully automated mass screening $[3,4]$. Such systems screen a large number of retinal images and identify abnormal images, which are then further examined by an ophthalmologist. This would save a significant amount of workload and time for ophthalmologists, allowing them to concentrate their resources on surgery and treatment. This paper explores a GFFNN classifier system which classify images into normal and abnormal images[5]. 64-point Discrete Cosine Transform (DCT) and 09 statistical parameters such as Entropy, Mean, Standard deviation, Average, Euler number, Contrast,
Correlation, Energy and Homogeneity are extracted from fundus retinal images to form a feature vector. This feature vector is used as an input to classifier system.

\section{DATABASE ACQUISITION}

A necessary tool for the reliable evaluation and comparison of medical image processing algorithms is a database including a selected set of high quality medical images which are representatives of diabetic retinopathy and have been verified by experts. For this work DIARETDB0 database was used. The current database consists of 130 colour fundus retinal images of which 20 are normal and 110 contain signs of the diabetic retinopathy (abnormal).

\section{FEATURE EXTRACTION}

Detection of retinopathy involves clinical recognition of dilation of blood vessels, presence of exudates, lesions or any other such abnormality in the retinal images. Fundus image not only provides anatomical information of the retina in eye, but also the information of these pathological features. In a healthy retinal image, the features observed are network of blood vessels, macula and the optic disc. Any change due to diabetic retinopathy or other disease will cause variation in these salient features which makes it easy to diagnose the disease. Fig. 1 shows a healthy gray level retinal image while fig. 2 shows the retinal image affected with diabetic retinopathy.

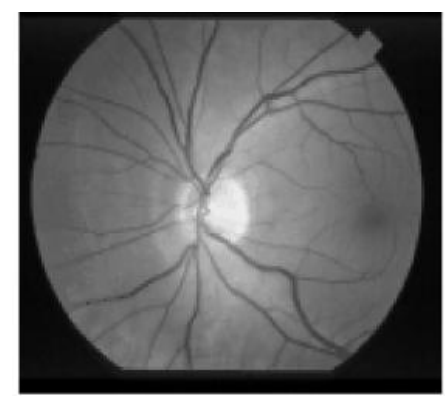

Fig. 1. Fundus Image of a healthy eye

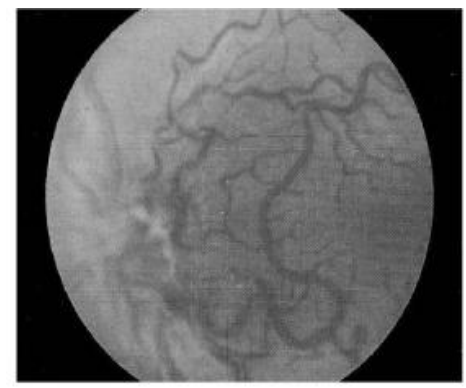

Fig. 2. Fundus image affected with diabetic retinopathy 


\section{GENERALISED FEED FORWARD NEURAL NETWORK}

Generalized feed forward networks are a generalization of the MLP such that connections can jump over one or more layers. In theory, a MLP can solve any problem that a generalized feed forward network can solve. In practice, however, generalized feed forward networks often solve the problem much more efficiently. A classic example of this is the two spiral problem. Without describing the problem, it suffices to say that a standard MLP requires hundreds of times more training epochs than the generalized feed forward network containing the same number of processing elements. In this neural network, each layer has an associated learning rule and learning parameters. The transfer functions those are available within the various Axon components. Each one of these axon components applies a static map to the data it receives. The map can be linear or nonlinear, or it can normalize the input to the PE. Back propagation is the most common form of learning. Fig. 3 shows GFFNN with two hidden layers \& three output layers.

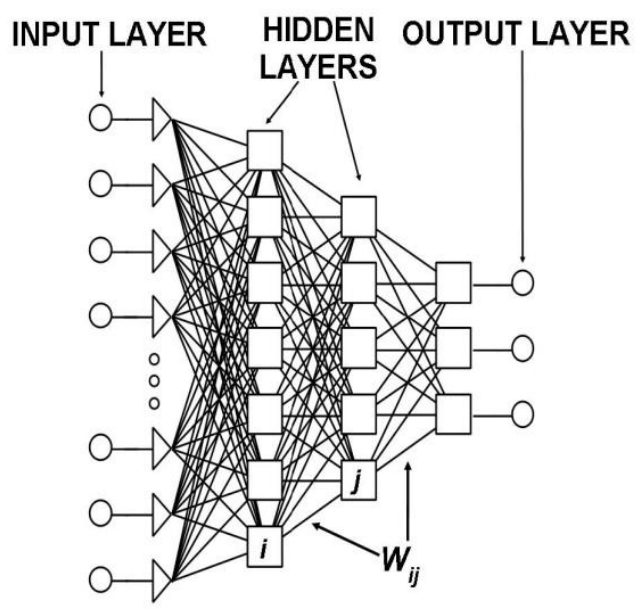

Fig. 3. Generalized feed forward network with two hidden layers \& one output layer

\section{OVERVIEW OF CLASSIFIER SYSTEM}

The authors employed GFFNN for the detection of diabetic retinopathy. The block diagram of the proposed system is shown in fig. 4. It consists of modules such as Retinal Fundus Image input, Pre-processing (decolour, resize), Feature Extraction(64 point DCT with statistical parameters), formation of feature optimize vector, GFFNN Classifier, Design of optimal GFFNN classifier by selecting hidden layers, learning rule, transfer function and step size etc. and Testing of Cross validation/Test dataset. Fundus retinal images are used as input for classifier system [6]. The extracted features vector of all images is first randomized and then fed to the GFFNN for training of neural network $[7,8,9]$. The GFFNN was used to test the proposed feature vector of retinal images to classify as normal and abnormal images.

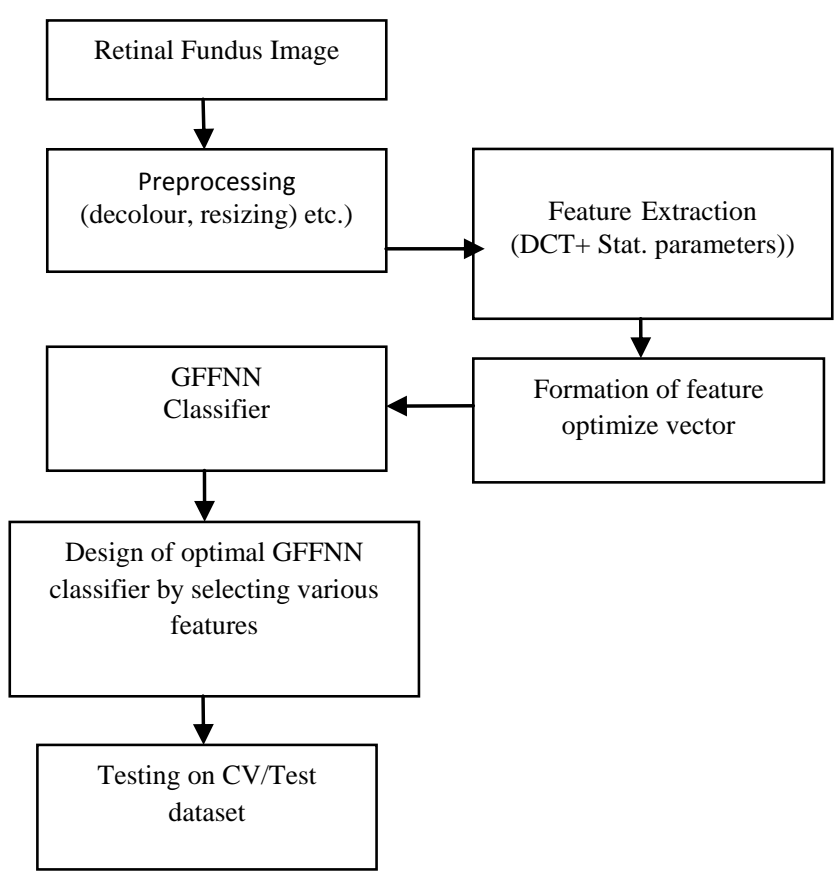

Fig. 4. Overview of classifier system

\section{RESULTS}

The GFFNN is designed using one hidden layer with single neuron. Then progressively numbers of neurons are increased and observed the results. Various parameters are changed progressively to set GFFNN with optimal results and least complexity. It is observed that with single hidden layer GFFNN gives better performance.

The number of Processing Elements (PEs) in the hidden layer also varied. The network is trained and final minimum Mean Square Error (MSE) is obtained on train and cross validation data sets when 03 PEs are used in the hidden layer as indicated in fig 5.

Final minimum MSE on training and CV data along with average classification accuracy is calculated and shown in fig. $6 \& 7$ for percentage of tagging data.

Various transfer functions are used for training the network and final minimum MSE on training and CV data is measured is shown in fig. 8. Percentage of average classification accuracy for different transfer functions is also shown in fig. 9.

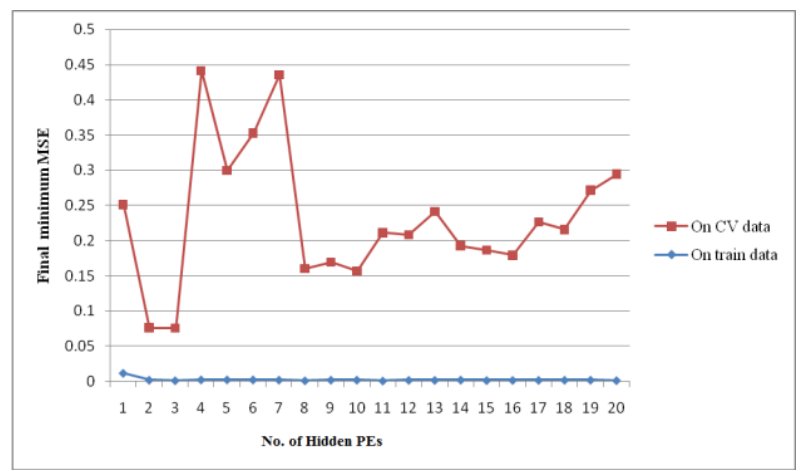

Fig. 5. : Graph showing variation of final minimum MSE with number of processing elements 


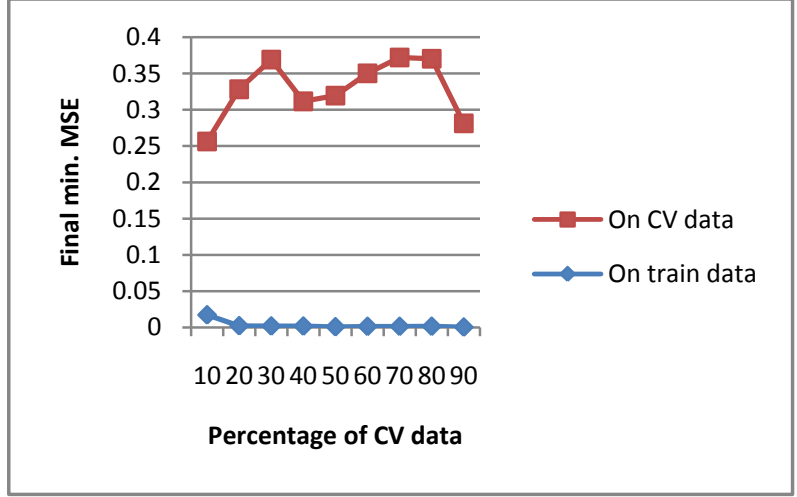

Fig. 6. : Graph indicating variation of final minimum MSE with \% of CV data.

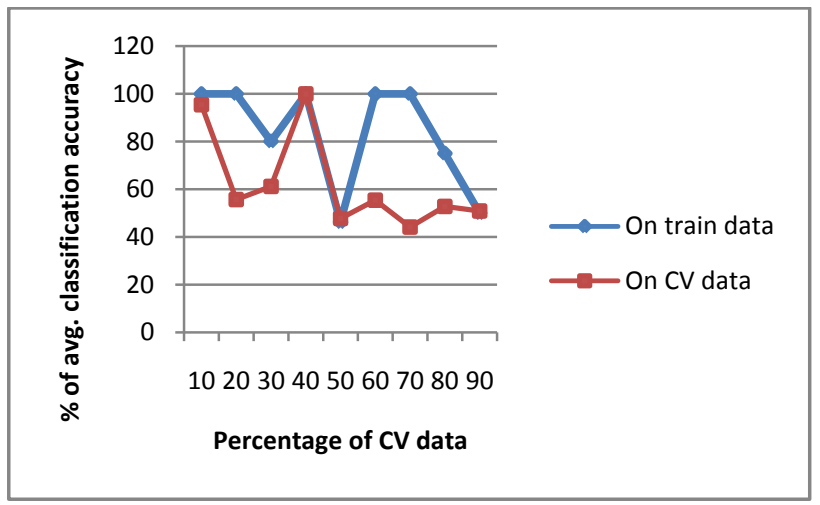

Fig. 7. : Graph showing variation of \% average classification accuracy with $\%$ of $\mathrm{CV}$ data

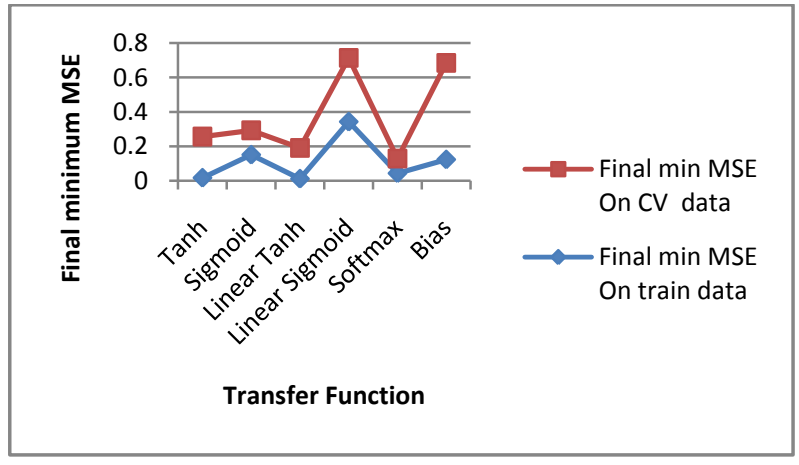

Fig. 8. : Graph showing variation of Final minimum MSE with different transfer functions

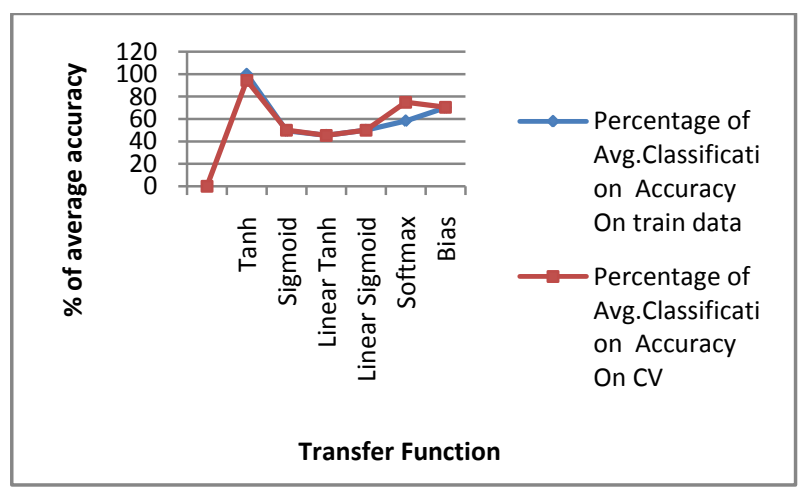

Fig. 9. : Graph showing variation of $\%$ of average classification accuracy with different transfer functions
With tanh transfer function the GFFNN neural network is trained using different learning rules. Final minimum MSE on training and $\mathrm{CV}$ data set is indicated in fig. 10 and percentage average classification accuracy is as shown in fig. 11.

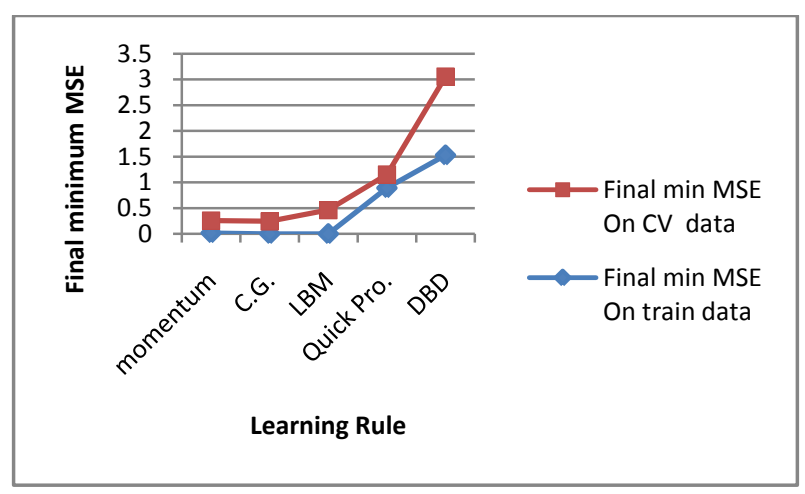

Fig. 10. : Graph showing variation of Final minimum MSE with learning rule

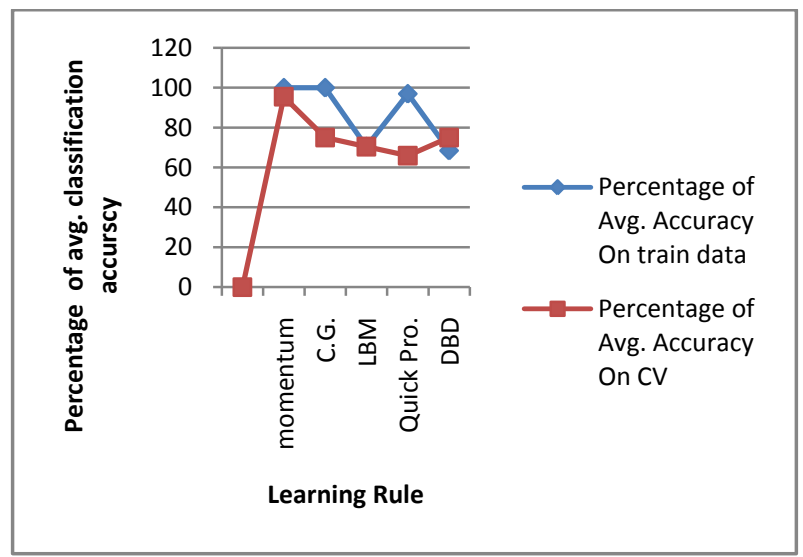

Fig. 11. : Graph showing variation of \% of average classification accuracy with learning rule

Various step size changed for training the network and final minimum MSE on training and CV data sets is measured is shown in fig.12. Percentage of average classification accuracy for different step size is also shown in fig.13.

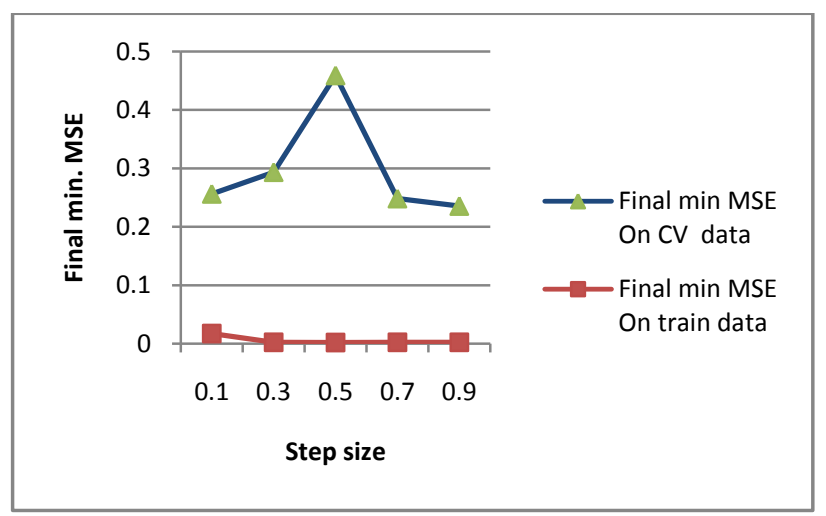

Fig. 12. : Graph showing variation of Final minimum MSE with different step size 


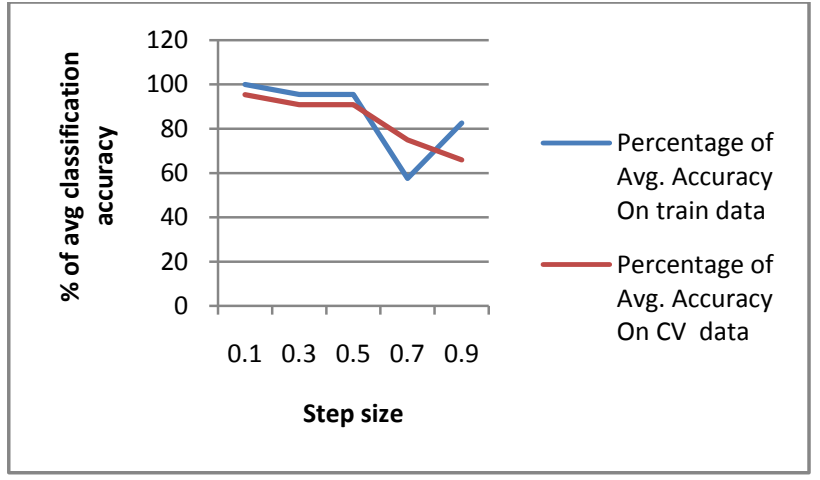

Fig. 13. : Graph showing variation of $\%$ of average classification accuracy with different step size

\subsection{The GFF-NN classifier Design}

\section{Parameters:}

The feature vector formed with 64-point DCT with 09 statistical parameters is fed to GFFNN as an input. The dataset is randomized. Training of classifier is done by varying the number of hidden layers, number of Neurons in a hidden layer, step size, learning rule, type of axon, number of epochs, number of runs, percentage of data tagging to obtain optimal selection of GFFNN as:

Tag data $: \mathrm{CV}=10 \%$ and train $=90 \%$

Exemplars $=117$

Hidden layer $=01$

Number of PEs $=03$

Transfer function $=\tanh A x o n$

Learning rule $=$ Momentum

Step size $=0.1$

Momentum $=0.7$

Output layer PEs. $=07$

Transfer function $=\tanh A x o n$

Learning rule $=$ Momentum

Step size $=0.1$

Momentum $=0.7$

Supervised learning control

Maximum epochs $=5000$

Termination - MSE increase, CV set

Weight update $=$ Batch

Training of Network

Number of epochs $=5000$

Number of runs $=03$

Termination is after 500 epochs without improvement.
Time elapsed per epoch per exemplar $=0.07 \mathrm{~ms}$ on Intel Celeron ${ }^{\circledR 2955 U}-1.40 \mathrm{GHz}$ computer system with $2 \mathrm{~GB}$ RAM along with 64 bit operating system.

Finally, designed GFFNN classifier is tested on training and $\mathrm{CV}$ datasets and results are shown in table 1 to 4 . Table $1 \& 2$ indicate the classification accuracy of training data is $100 \%$ for normal and abnormal images.

Table 1: confusion matrix on training data

\begin{tabular}{|l|c|c|}
\hline Output / Desired & o1(Abnormal) & o2(Normal) \\
\hline o1(Abnormal) & 99 & 0 \\
\hline o2(Normal) & 0 & 18 \\
\hline
\end{tabular}

Table2: Performance parameters for training data

\begin{tabular}{|c|c|c|}
\hline Performance & o1(Abnormal) & o2(Normal) \\
\hline MSE & 0.00078275 & 0.00080599 \\
\hline NMSE & 0.006012942 & 0.006191472 \\
\hline MAE & 0.020048299 & 0.020156657 \\
\hline Min Abs Error & $1.1093 \mathrm{E}-05$ & $9.3374 \mathrm{E}-05$ \\
\hline Max Abs Error & 0.0555492 & 0.055547759 \\
\hline R & 0.997831718 & 0.997818887 \\
\hline Percent Correct & 100 & 100 \\
\hline
\end{tabular}

The overall accuracy is $=100 \%$

Table 1: confusion matrix on $\mathrm{CV}$ data

\begin{tabular}{|l|c|c|}
\hline Output / Desired & o1(Abnormal) & o2(Normal) \\
\hline o1(Abnormal) & 10 & 0 \\
\hline o2(Normal) & 1 & 2 \\
\hline
\end{tabular}

Table2: Performance parameters for $\mathrm{CV}$ data

\begin{tabular}{|c|c|c|}
\hline Performance & o1(Abnormal) & o2(Normal) \\
\hline MSE & 0.043584561 & 0.04800291 \\
\hline NMSE & 0.334808674 & 0.368749627 \\
\hline MAE & 0.13885197 & 0.127054878 \\
\hline Min Abs Error & 0.005363616 & 0.008494312 \\
\hline Max Abs Error & 0.509371244 & 0.655460425 \\
\hline R & 0.818011051 & 0.818251707 \\
\hline Percent Correct & 90.90909091 & 100 \\
\hline
\end{tabular}

The overall accuracy is $=95.45 \%$ 
The overall accuracy of detection of diabetic retinopathy is $100 \%$ and 95.45 for normal and abnormal retinal images on train data and cross validation data sets respectively.

\section{DISCUSSION AND CONCLUSION}

This paper proposed GFFNN classifier system for detection of diabetic retinopathy in retinal images. Different features like 64-point DCT along with statistical parameters like Entropy, mean, standard deviation, average, Euler number, contrast, correlation, energy and homogeneity are extracted from retinal images. These features formed the feature vector which is used as input to the classifier. The Train $\mathrm{N}$ Times training method is used to train the GFFNN classifier. Designed GFFNN classifier achieved high \% of classification accuracy of $100 \%$ for training data and $95.45 \%$ for cross validation data sets.

\section{REFERENCES}

[1] Meindert Niemeijer,"Retinopathy Online Challenge: Automatic Detection of Microaneurysms in Digital Color Fundus Photographs",IEEE Transactions on Medical Imaging vol.29,no.1, January 2010..

[2] D. Klein, B. E Klein, S. E Moss et al "The Wisconsin epidemiologic study of diabetic retinopathy VII.Diabetic non proliferative retinal lesions", Br. J Ophthalmol, vol. 94, 1986.

[3] Michael Goldbaum,Saied Moezzi, et al., "Automated diagnosis and image understanding with object extraction, object classification, and differencing in retinal images”,. Br. J Ophthalmol, vol 83, august 1999.
[4] C.Sinthanayothin, J.Boyce,et al.,"Automated localisation of optic disc, fovea, and retinal blood vessels from digital colour fundus images", Br. J Ophthalmol, vol. 83, august 1999.

[5] Anil K. Jain Michigan State University, Jianchang Mao IBM Almaden Research Centre.," Artificial neural networks: A tutorial",1996.

[6] S.JeraldJeba Kumar, Madheswaran, "Extraction of Blood Vascular Network for Development of an Automated Diabetic Retinopathy Screening System", International Conference on Computer Technology and Development IEEE 10.1109/Icctd.2009.212, 2009.

[7] María García, Carmen Valverde, "Comparison of Logistic Regression and Neural Network Classifiers in the Detection of Hard Exudates in Retinal Images", 35th Annual International Conference of the IEEE EMBS Osaka, Japan, 3 - 7 July, 2013.

[8] G.U.Kharat \& S.V.Dudul," Optimal Neural Network Classifier for Human Emotion Recognition from Facial Expression using Singular Value Decomposition (SVD)", Internal journal of Engineering Research and Industrial Application(IJERIA),pp 155-166,vol I,No.04,(ISSN-09704-1518),2008.

[9] A.P.Bhatkar \& G.U.Kharat," Detection of Diabetic Retinopathy in Retinal Images using MLP classifier ", 2015 IEEE International Symposium on Nanoelectronic and Information Systems, 978-1-4673-9692-9/15 \$31.00 (c) 2015 IEEE DOI 10.1109/iNIS.2015.30. 